\title{
Uma sequência infinita de radicais encaixados
}

Élis Gardel Mesquita(D)

\author{
Thiago R. Cavalcante (D)
}

\section{Resumo}

Neste trabalho fazemos um estudo sobre a classe de sequências definidas por radicais encaixados da forma $\left(x_{n}\right)_{n}=(\sqrt[q]{a}, \sqrt[q]{a+\sqrt[q]{a}}, \cdots)$. Tal sequência obedece à relação de recorrência não linear $x_{n}(a)=\sqrt[q]{a+x_{n-1}(a)}, c o m x_{0}(a)=\sqrt[q]{a}$. Utilizando um teorema clássico do estudo de sequências, resolvemos o problema da convergência dessa sequência para determinados valores de a e q. Em seguida, explorando a equação característica associada, dada por $\mathrm{x}^{\mathrm{q}}-\mathrm{x}-\mathrm{a}=0$, através de uma técnica de interseção de gráficos, obtemos expressões explícitas para o limite.

Palavras-chave: Radicais encaixados, Recorrência não linear, Sequência de números reais.

In this work, we study a class of sequences defined by nested radicals of the form $\left(x_{n}\right)_{n}=(\sqrt[q]{a}$, $\sqrt[q]{a+\sqrt[q]{a}}, \cdots)$. This sequence satisfies the nonlinear recurrence relation $x_{n}(a)=\sqrt[q]{a+x_{n-1}(a)}$, with $\mathrm{x}_{0}(\mathrm{a})=\sqrt[q]{\mathrm{a}}$. Using a classic Theorem about sequences, we solve the convergence problem of this sequence for a certain values of a e q. Moreover, by the analysis of the associated characteristic equation, given by $\mathrm{x}^{\mathrm{q}}-\mathrm{x}-\mathrm{a}=0$, through graphics intersection technique, we obtain explicit expressions to the limit.

keywords: Nested radicals, Nonlinear recurrence, Sequence of real numbers.

\section{Introdução}

Uma grande variedade de problemas, das diversas áreas do conhecimento, decaem no estudo de sequências de números reais. Um tipo de sequência bem conhecida e que desperta a curiosidade de muitos matemáticos são aquelas definidas através de múltiplas composições de radicais, as chamadas de sequência de radicais encaixados. Grandes matemáticos dedicaram tempo para tentar entender melhor esse tipo de sequência. Aqui iremos mencionar dois deles.

O primeiro a que nos referimos é o matemático francês François Viète (1540 - 1603). Este trabalhou com sequências infinitas de radicais encaixados dentre as quais duas são bastante conhecidas nos dias atuais, $\left(\mathrm{x}_{\mathrm{n}}\right)_{\mathrm{n}}=(\sqrt{2}, \sqrt{2+\sqrt{2}}, \cdots)$ e $\left(\mathrm{y}_{\mathrm{n}}\right)_{\mathrm{n}}=(\sqrt{2}, \sqrt{2-\sqrt{2}}, \sqrt{2-\sqrt{2+\sqrt{2}}}, \cdots)$. A razão para isso é que tais sequências estão relacionadas com o número irracional $\pi$ e tal relação é estabelecida através dos limites

$$
\frac{2}{\pi}=\frac{\sqrt{2}}{2} \cdot \frac{\sqrt{2+\sqrt{2}}}{2} \cdot \frac{\sqrt{2+\sqrt{2+\sqrt{2}}}}{2} \cdot \ldots \quad \text { e } \quad 2^{\mathrm{k}} \quad \frac{\sqrt{2-\sqrt{2+\sqrt{2+\cdots+\sqrt{2}}}}}{\mathrm{k} \text { vezes }} \rightarrow \frac{\pi}{2} .
$$


Essas identidades são conhecidas como fórmulas de Viète. Para mais detalhes e outras informações sugerimos [1, página 154], [4, 5, 6, 10, 12] e referências contidas neles.

O segundo é o famoso matemático indiano Srinivasa Ramanujan (1887-1920), o qual publicou um desafio no importante Journal of the Indian Mathematical Society: determinar o valor da expressão dada em radicais encaixados:

$$
\sqrt{1+2 \sqrt{1+3 \sqrt{ } \cdots}}
$$

Após esperar por aproximadamente seis meses sem sucesso, ele mesmo forneceu uma equação que resolve o problema:

$$
x+n+a=\sqrt{a x+(n+a)^{2}+x \sqrt{a(x+n)+(n+a)^{2}+(x+n) \sqrt{\cdots}}}
$$

Nesse caso, tomando $\mathrm{x}=2, \mathrm{n}=1$ e $\mathrm{a}=0$, vemos que

$$
3=2+1+0=\sqrt{0 \cdot 2+(1+0)^{2}+2 \sqrt{0(2+1)+(1+0)^{2}+(2+1) \sqrt{\cdots}}},
$$

Ou seja, 3 é a solução para expressão. Para mais detalhes e outras curiosidades sobre Ramanujan e radicais encaixados, sugerimos [9,11]. Esse mesmo problema sugerido por Ramanujan aparece no banco de questões da Obmep, Olimpíadas Brasileira de Matemática das Escolas Públicas, de 2018, questão 22 item b), como se pode conferir em [3], revelando assim o papel pedagógico do assunto.

Várias outras questões sobre radicais encaixados são exploradas em olimpíadas de matemática, como a questão 6 do nível 3 do banco de questões de 2016 da Obmep, como podemos ver em [2], na qual se pede para encontrar o valor da expressão dada em radicais encaixados

$$
\sqrt{1+2014 \sqrt{1+2015 \sqrt{1+2016 \cdot 2018}}}
$$

A solução é obtida utilizando a identidade $(n-1)(n+1)+1=n^{2}$. Com isso,

$$
\begin{aligned}
\sqrt{1+2014 \sqrt{1+2015 \sqrt{1+2016 \cdot 2018}}} & =\sqrt{1+2014 \sqrt{1+2015 \sqrt{2017^{2}}}} \\
& =\sqrt{1+2014 \sqrt{1+2015 \cdot 2017}} \\
& =\sqrt{1+2014 \sqrt{2016^{2}}} \\
& =\sqrt{1+2014 \cdot 2016} \\
& =\sqrt{2015^{2}} \\
& =2015 .
\end{aligned}
$$


Neste trabalho, estamos particularmente interessados em estudar uma generalização da sequência de radicais encaixados do tipo $\left(\mathrm{x}_{\mathrm{n}}\right)_{\mathrm{n}}=(\sqrt{2}, \sqrt{2+\sqrt{2}}, \cdots)$. Tal generalização é obtida trocando o índice do radical 2 por um número inteiro $\mathrm{q} \geq 2$ e o número 2 no radicando por um número real positivo qualquer. Ou seja, vamos estudar a sequência infinita de radicais encaixados generalizada da forma

$$
\left(x_{n}(a, q)\right)_{n}=(\sqrt[q]{a}, \sqrt[q]{a+\sqrt[q]{a}}, \cdots) .
$$

Zimmerman e Ho, em [13], fizeram um estudo detalhado de algumas sequências de radicais encaixados que inclui a sequência $\left(\mathrm{x}_{\mathrm{n}}\right)_{\mathrm{n}}$ e deram um passo na direção que pretendemos seguir. Generalizando o radicando, trocando 2 por um número real positivo a, obtendo assim $\left(\mathrm{x}_{\mathrm{n}}(\mathrm{a})\right)_{\mathrm{n}}=$ $(\sqrt{a}, \sqrt{a+\sqrt{a}}, \cdots)$, eles demonstraram que a sequência é convergente e obtiveram a seguinte expressão para o limite

$$
\sqrt{a+\sqrt{a+\sqrt{a+\cdots}}}=\frac{1+\sqrt{1+4 a}}{2} \text {. }
$$

Vale destacar o curioso fato que segue da equação acima. O número de ouro $(1+\sqrt{5}) / 2$, pode ser expresso como o limite de uma sequência de radicais encaixados, ou seja,

$$
\sqrt{1+\sqrt{1+\sqrt{1+\cdots}}}=\frac{1+\sqrt{5}}{2} .
$$

Uma das grandes dificuldades no estudo de sequências de radicais encaixados é a obtenção de expressões simples para o limite. Dado que temos a expressão para o limite no caso do radical de índice 2, podemos tentar simplificá-la ainda mais. Por exemplo, se $\mathrm{r}$ um número racional maior que 1 e tomando $\mathrm{a}=\mathrm{r}(\mathrm{r}-1)$ na equação 2 , obtemos

$$
\sqrt{r(r-1)+\sqrt{r(r-1)+\cdots}}=r
$$

a qual é uma expressão relativamente simples. Com isso, a equação acima fornece uma maneira de se obter exemplos de números racionais que podem ser escritos como limite de sequência infinita de radicais encaixados.

Herschfeld, [6], estudou a sequência $\left(x_{n}(a)\right)_{n}=(\sqrt{a}, \sqrt{a+\sqrt{a}}, \cdots)$ utilizando outros métodos para demonstrar a convergência, e apresenta uma grande variedade de maneiras de como obter expressões bastante simples para o limite. Por exemplo,

$$
\sqrt{2^{2}+\frac{r}{2} \sqrt{2^{4}+\frac{r}{2} \sqrt{2^{6}+\cdots}}}=2\left(1+\frac{r}{4}\right)
$$

$\mathrm{e}$

$$
\sqrt{1+\frac{r}{2} \sqrt{1+\frac{r}{2^{2}} \sqrt{1+\cdots+\sqrt{1+\frac{r}{2^{n}} \sqrt{\cdots}}}}}=1+\frac{r}{4} .
$$


O que está por trás das descobertas acima é a relação entre as raízes de uma equação quadrática, a saber $\mathrm{x}^{2}-\mathrm{x}-\mathrm{a}=0$, e o limite da sequência. No entanto, quando generalizamos o índice do radical, somos levados a estudar as raízes positivas da equação mais geral

$$
\mathrm{x}^{\mathrm{q}}-\mathrm{x}-\mathrm{a}=0
$$

que, como sabemos, não é uma tarefa fácil.

Nossos objetivos são:

- fornecer condições em a e q de maneira a assegurar a convergência da sequência de radicais encaixados generalizada (1);

- demonstrar que o limite de $\left(x_{n}(a, q)\right)_{n}$ possui apenas $\sqrt[q-1]{2}$ como ponto fixo, generalizando nesse sentido o Teorema 2 atribuído a Viète, ou seja, para todo inteiro q $\geq 2$ vale

$$
\sqrt[q]{q-1 \sqrt{2}+\sqrt[q]{\frac{q-1}{2}+\sqrt[q]{q-1} \sqrt{2}+\cdots}}=\sqrt[q-1]{2}
$$

- estabelecer relações entre o termo que aparece no radicando e o limite da sequência, que será umas das possíveis raízes da equação (4), com intuito de obtermos formas explícitas de escrever números reais como limite de sequências de radicais encaixados. Ou seja, estendemos resultados em $[6,13]$ provando que para todo inteiro $q \geq 2$ e todo real $r>0$ vale

$$
\sqrt[q]{r^{q-1} \sqrt{r+1}+\sqrt[q]{r^{q-1} \sqrt{r+1}+\sqrt[q]{r^{q-1} \sqrt{r+1}+\cdots}}}=\sqrt[q-1]{r+1}
$$

O diagrama abaixo resume as generalizações de $\left(x_{n}\right)_{n}=(\sqrt{2}, \sqrt{2+\sqrt{2}}, \cdots)$.

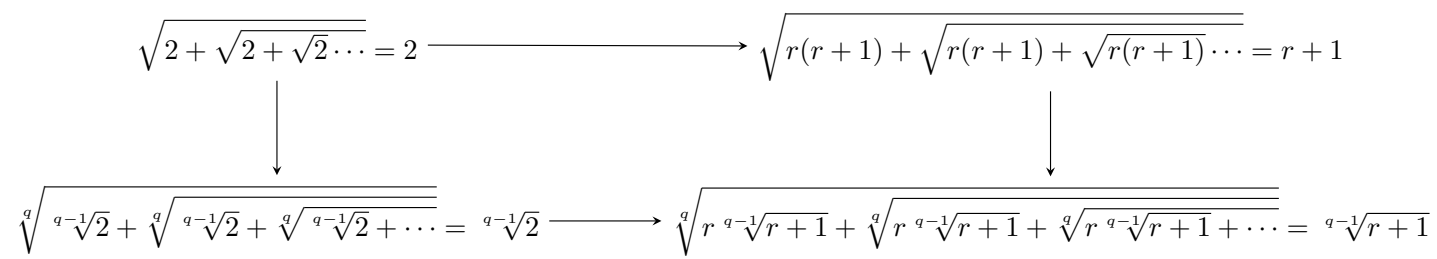

\section{Discussão preliminar}

O resultado básico que vamos utilizar é um teorema clássico do estudo de sequências de números reais qual, por completeza, será enunciado abaixo e o mesmo pode ser encontrado em [7, página 111]. Além desse Teorema, esperamos que o leitor tenha uma noção intuitiva de indução finita e do conceito de limite de sequência. 
Teorema 1. Toda sequência monótona limitada é convergente.

O teorema a seguir, atribuído a Viète, é o resultado que iremos estender em alguns sentidos, neste trabalho.

Teorema 2. A sequência $\left(\mathrm{x}_{\mathrm{n}}\right)_{\mathrm{n}}=(\sqrt{2}, \sqrt{2+\sqrt{2}}, \sqrt{2+\sqrt{2+\sqrt{2}}}, \ldots)$ é convergente, e o limite é 2 .

Demonstração. Note que, como $\sqrt{2}>0$, então $\mathrm{x}_{1}=\sqrt{2}<\sqrt{2+\sqrt{2}}=\mathrm{x}_{2}$. Utilizaremos indução sobre $\mathrm{n}$ para provar que a sequência $\mathrm{x}_{\mathrm{n}}$ é crescente e portanto monótona. Temos que $\mathrm{x}_{1}<\mathrm{x}_{2}$, como descrito anteriormente. Iremos supor que $\mathrm{x}_{\mathrm{n}}<\mathrm{x}_{\mathrm{n}+1}$ e provaremos que $\mathrm{x}_{\mathrm{n}+1}<\mathrm{x}_{(\mathrm{n}+1)+1}$, garantindo assim o crescimento da sequência. Utilizando a definição da sequência, dada pela recorrência $\mathrm{x}_{\mathrm{n}}=\sqrt{2+\mathrm{x}_{\mathrm{n}-1}}$, com $\mathrm{x}_{0}=\sqrt{2}$, a hipótese de indução $\mathrm{x}_{\mathrm{n}}<\mathrm{x}_{\mathrm{n}+1}$ e o fato de a função raiz quadrada ser monótona crescente, temos que

$$
\mathrm{x}_{\mathrm{n}+1}=\sqrt{2+\mathrm{x}_{\mathrm{n}}}<\sqrt{2+\mathrm{x}_{\mathrm{n}+1}}=\mathrm{x}_{\mathrm{n}+2} \text {, }
$$

concluindo assim que $\mathrm{x}_{\mathrm{n}}$ é crescente. Devido ao seu crescimento, a sequência $\mathrm{x}_{\mathrm{n}}$ é limitada inferiormente por $\mathrm{x}_{0}=\sqrt{2}$. Nesse momento, novamente utilizando indução sobre n, vamos provar que $\mathrm{x}_{\mathrm{n}}$ é limitada superiormente por 2 e concluir que se trata de uma sequência limitada. Temos que $\mathrm{x}_{0}=\sqrt{2}<2, \mathrm{x}_{1}=\sqrt{2+\sqrt{2}}<\sqrt{2+2}=2$. Suponha que $\mathrm{x}_{\mathrm{n}}<2$. Por definição $\mathrm{x}_{\mathrm{n}+1}=\sqrt{2+\mathrm{x}_{\mathrm{n}}}<\sqrt{2+2}=2$. Portanto $\left(\mathrm{x}_{\mathrm{n}}\right)_{\mathrm{n}}$ é limitada superiormente por 2 .

$\mathrm{Na}$ verdade podemos calcular explicitamente tal limite através da relação de recorrência. Denote por x o limite da sequência $\left(x_{n}\right)_{n}, x=\lim x_{n}$. Aplicando o limite em ambos os lados da equação $\mathrm{x}_{\mathrm{n}}=\sqrt{2+\mathrm{x}_{\mathrm{n}-1}}$, e eliminando o radical, obtemos a equação quadrática $\mathrm{x}^{2}-\mathrm{x}-2=0$ cujas raízes são $\mathrm{x}=-1$ e $\mathrm{x}=2$. Como $\mathrm{x}$ deve ser não negativo, devemos ter $\mathrm{x}=2$.

Ao substituirmos 2 por um número real positivo qualquer a, temos o

Teorema 3 (Zimmerman-Ho). Para todo número real a $>$ 0, a sequência de radicais encaixados $\left(\mathrm{x}_{\mathrm{n}}(\mathrm{a})\right)_{\mathrm{n}}=(\sqrt{\mathrm{a}}, \sqrt{\mathrm{a}+\sqrt{\mathrm{a}}}, \sqrt{\mathrm{a}+\sqrt{\mathrm{a}+\sqrt{\mathrm{a}}}}, \ldots)$ é convergente.

Demonstração. A demonstração é análoga à demonstração do Teorema anterior e pode ser encontrada em [13], Lema 1.

O limite de $\left(\mathrm{x}_{\mathrm{n}}(\mathrm{a})\right)_{\mathrm{n}}$, por sua vez, é dado pela raiz positiva da equação quadrática $\mathrm{x}^{2}-\mathrm{x}-\mathrm{a}=0$, ou seja, se x(a) denota tal limite, então

$$
\mathrm{x}(\mathrm{a})=\frac{1+\sqrt{1+4 \mathrm{a}}}{2}
$$

Vale destacar que, para $\mathrm{a}>0$, as raízes da equação $\mathrm{x}^{2}-\mathrm{x}-\mathrm{a}=0$ possuem sinais contrários. De fato, a segunda raiz é dada por

$$
\overline{x(a)}=\frac{1-\sqrt{1+4 a}}{2},
$$


e, como podemos ver, ela é positiva apenas para $-\frac{1}{4}<\mathrm{a}<0$, o que contrariaria a hipótese do Teorema 3.

O próximo resultado fornece uma forma de escrever números racionais como sequência infinita de radicais encaixados. Mais especificamente, dado um racional $\mathrm{r}>1$, qual deve ser o valor do racional a para o qual se tenha $\mathrm{x}(\mathrm{a})=\mathrm{r}$ ? A resposta é encontrada no resultado seguinte.

Teorema 4 (Zimmerman-Ho). Dado um número racional $\mathrm{r}>1$, existe um único número racional $\mathrm{a}=\mathrm{r}(\mathrm{r}-1)$ tal que $\mathrm{x}(\mathrm{a})=\mathrm{r}$.

Demonstração. Com efeito, dado $\mathrm{r}>1$ faça $\mathrm{a}=\mathrm{r}(\mathrm{r}-1)$ em (5). Com isso, obtemos

$$
\mathrm{x}(\mathrm{r}(\mathrm{r}-1))=(1+\sqrt{1+4 \mathrm{r}(\mathrm{r}-1)}) / 2=\left(1+\sqrt{1+4 \mathrm{r}^{2}-4 \mathrm{r}}\right) / 2=\left(1+\sqrt{(2 \mathrm{r}-1)^{2}}\right) / 2=(2 \mathrm{r}) / 2=\mathrm{r}
$$

Observação 1. A notação clássica para o limite de $\left(\mathrm{x}_{\mathrm{n}}(\mathrm{a})\right)_{\mathrm{n}}$ é

$$
\lim _{n \rightarrow \infty} x_{n}(a)=\sqrt{a+\sqrt{a+\sqrt{a+\cdots}}}
$$

Aqui iremos usar a notação análoga, ou seja, o limite da sequência generalizada $\left(x_{n}(a, q)\right)_{n}$ é denotado por

$$
\lim _{n \rightarrow \infty} x_{n}(a, q)=\sqrt[q]{a+\sqrt[q]{a+\sqrt[q]{a+\cdots}}}
$$

Por exemplo, aplicando o Teorema 4, temos:

$$
\begin{aligned}
3 & =\sqrt{6+\sqrt{6+\sqrt{6+\cdots}}}, \text { onde } a=6=3(3-1), \\
6 & =\sqrt{30+\sqrt{30+\sqrt{30+\cdots}}}, \text { onde } a=30=6(6-1) .
\end{aligned}
$$

Observação 2. Observe que, se pensarmos em uma fração $\mathrm{r}=\frac{\mathrm{x}}{\mathrm{y}}>1$ arbitrária, obtemos

$$
r=\frac{x}{y} \Rightarrow a=\frac{x}{y}\left(\frac{x}{y}-1\right)
$$

De onde teríamos

$$
\frac{x}{y}=\sqrt{\frac{x^{2}-x y}{y^{2}}+\sqrt{\frac{x^{2}-x y}{y^{2}}+\sqrt{\frac{x^{2}-x y}{y^{2}}+\cdots}}}
$$

Por exemplo

$$
\frac{3}{2}=\sqrt{\frac{3}{4}+\sqrt{\frac{3}{4}+\sqrt{\frac{3}{4}+\cdots}}}, \quad \text { onde } \mathrm{a}=\frac{3}{4}=\frac{3}{2}\left(\frac{3}{2}-1\right) .
$$

Na Seção 4 vamos obter um resultado deste tipo quando o índice do radical é maior ou igual a 2 e o termo no argumento a que aparece no radicando é permitido ser irracional.

Definição 1. Um número real a é dito um ponto fixo de uma função $\mathrm{f}: \mathbb{R} \rightarrow \mathbb{R}$ se vale $\mathrm{f}(\mathrm{a})=\mathrm{a}$. 
O leitor pode verificar que $\mathrm{x}(\mathrm{a})=\mathrm{a}$ se, e somente $\mathrm{se}, \mathrm{a}=2$. Ou seja, quando $\mathrm{q}=2$, o ponto fixo da função a $\mapsto \mathrm{x}(\mathrm{a})$, que associa a cada real positivo a a raiz positiva da equação $\mathrm{x}^{2}-\mathrm{x}-\mathrm{a}=0$, é o número 2. E se fixarmos um inteiro $q \geq 2$, o ponto fixo ainda é único? qual o seu valor? mais sucintamente, surge a seguinte questão:

Questão 1. Fixado um inteiro $q \geq 2$, para quais valores do argumento $a=a(q)$ no radicando a sequência de radicais encaixados $\left(x_{n}(a)\right)_{n}=(\sqrt[q]{a}, \sqrt[q]{a+\sqrt[q]{a}}, \ldots)$ satisfaz $\sqrt[q]{a+\sqrt[q]{a+\cdots}}=a$ ?

Para responder a essa questão, vamos generalizar o Teorema 3 trocando o índice do radical 2 por um número inteiro qualquer $q \geq 2$.

\section{Resultado Principal}

Nesta seção vamos reescrever a sequência de radicais encaixados generalizada usando a relação de recorrência $x_{n}(a, q)=\sqrt[q]{a+x_{n-1}(a, q)}, x_{0}(a, q)=\sqrt[q]{a}$. A demonstração da convergência segue de perto as generalizações dadas em [13] e a abordagem histórica do problema, isto é, utilizando indução para obter crescimento e limitação. Ao leitor que deseja obter outras generalizações e fórmulas que utilizam radicais encaixados e outros tipos de recorrências sugerimos, por exemplo, $[8]$.

Lema 1. Para qualquer a $\in \mathbb{R}_{+}^{*}$ e todo inteiro $\mathrm{q} \geq 2$, tem-se que $\mathrm{a}^{1 / \mathrm{q}}<2 \mathrm{e}^{\mathrm{a}}$, onde $\mathbb{R}_{+}^{*}$ denota os números reais estritamente positivos.

Demonstração. Segue da definição da função logarítmica e do fato q $\geq 2$, que a $>\ln a>\frac{1}{q} \ln a$, para todos a $\in \mathbb{R}_{+}^{*}$. Utilizando que a função exponencial que trabalhamos aqui é crescente e contínua, concluímos que

$$
2 \mathrm{e}^{\mathrm{a}}>\mathrm{e}^{\frac{1}{\mathrm{q}} \ln \mathrm{a}}=\mathrm{e}^{\ln \mathrm{a}^{\frac{1}{\mathrm{q}}}}=\mathrm{a}^{\frac{1}{\mathrm{q}}},
$$

donde segue o resultado.

Lema 2. A sequência dada pela recorrência $\mathrm{x}_{\mathrm{n}}(\mathrm{a}, \mathrm{q})=\sqrt[\mathrm{q}]{\mathrm{a}+\mathrm{x}_{\mathrm{n}-1}(\mathrm{a}, \mathrm{q})}, \operatorname{com} \mathrm{x}_{0}(\mathrm{a}, \mathrm{q})=\sqrt[q]{\mathrm{a}}$, é limitada.

Demonstração. A demonstração será feita por indução sobre n. De fato, pelo Lema 1

$$
\begin{aligned}
& x_{0}(a, q)=\sqrt[q]{a}<2 e^{a} \\
& x_{1}(a, q)=\sqrt[q]{a+\sqrt[q]{a}}<\sqrt[q]{e^{a}+2 e^{a}}<\sqrt[q]{3 e^{a}}<2 e^{a} .
\end{aligned}
$$

Suponha que $\mathrm{x}_{\mathrm{n}}(\mathrm{a}, \mathrm{q})<2 \mathrm{e}^{\mathrm{a}}$. Então, como a raiz q-ésima é uma função crescente para $\mathrm{q} \geq 2$, segue que $x_{n+1}(a, q)=\sqrt[q]{a+x_{n}(a, q)}<\sqrt[q]{a+2 e^{a}}<\sqrt[q]{3 e^{a}}<2 e^{a}$ e o resultado segue por indução.

O leitor pode verificar os passos omitidos: $\sqrt[q]{3}<2$ e $\sqrt[q]{\mathrm{e}^{\mathrm{a}}}<\mathrm{e}^{\mathrm{a}}$ para todo a $>0$ e todo inteiro $\mathrm{q} \geq 2$.

Lema 3. A sequência dada pela recorrência $\mathrm{x}_{\mathrm{n}}(\mathrm{a}, \mathrm{q})=\sqrt[\mathrm{q}]{\mathrm{a}+\mathrm{x}_{\mathrm{n}-1}(\mathrm{a}, \mathrm{q})}$, com $\mathrm{x}_{0}(\mathrm{a}, \mathrm{q})=\sqrt[\mathrm{q}]{\mathrm{a}}$, é crescente.

Demonstração. Temos que $\mathrm{x}_{0}(\mathrm{a}, \mathrm{q})=\sqrt[\mathrm{q}]{\mathrm{a}}<\sqrt[q]{\mathrm{a}+\sqrt[q]{\mathrm{a}}}=\mathrm{x}_{1}(\mathrm{a}, \mathrm{q})$. Suponha que $\mathrm{x}_{\mathrm{n}}(\mathrm{a}, \mathrm{q})<\mathrm{x}_{\mathrm{n}+1}(\mathrm{a}, \mathrm{q})$. Então $x_{n+1}(a, q)=\sqrt[q]{a+x_{n}(a, q)}<\sqrt[q]{a+x_{n+1}(a, q)}=x_{n+2}(a, q)$. Logo, o resultado segue por indução. 
Agora vamos enunciar o resultado principal de nosso estudo.

Teorema 5. Para todo a $\in \mathbb{R}_{+}^{*}$ e todo inteiro $\mathrm{q} \geq 2$, a sequência dada pela recorrência $\mathrm{x}_{\mathrm{n}}(\mathrm{a}, \mathrm{q})=$ $\sqrt[q]{\mathrm{a}+\mathrm{x}_{\mathrm{n}-1}(\mathrm{a})}$, com $\mathrm{x}_{0}(\mathrm{a}, \mathrm{q})=\sqrt[q]{\mathrm{a}}$, é convergente.

Demonstração. De fato segue dos Lemas 2 e 3 que a sequência de radicais generalizada é monótona e limitada. Portanto, convergente conforme o Teorema 1.

Dado um número real positivo a e um inteiro $q \geq 2$, seja $\mathrm{L}_{\mathrm{a}, \mathrm{q}}$ o limite da sequência $\left(\mathrm{x}_{\mathrm{n}}(\mathrm{a}, \mathrm{q})\right)_{\mathrm{n}}$. Então podemos determinar $\mathrm{L}_{\mathrm{a}, \mathrm{q}}$ resolvendo a equação

$$
\mathrm{x}^{\mathrm{q}}-\mathrm{x}-\mathrm{a}=0
$$

Corolário 1. Para todo inteiro $\mathrm{q} \geq 2$ e $\mathrm{a} \in \mathbb{R}_{+}^{*}$, a equação $\mathrm{x}^{\mathrm{q}}-\mathrm{x}-\mathrm{a}=0$ possui somente uma raiz positiva.

Demonstração. Suponha que $\mathrm{x}_{1}(\mathrm{a}, \mathrm{q})$ e $\mathrm{x}_{2}(\mathrm{a}, \mathrm{q})$ sejam raízes positivas da equação (6). Então, segue do Teorema 5 e da unicidade do limite que $x_{1}(a, q)=\lim _{n \rightarrow \infty} x_{n}(a, q)=x_{2}(a, q)$, ou seja, $x_{1}(a, q)=x_{2}(a, q)$.

\section{Equação característica e Aplicações}

Nesta seção vamos explorar a equação característica associada à recorrência a fim de obter exemplos e expressões explícitas para o limite da sequência de radicais encaixados.

Definição 2 (Equação característica). Dados q e a como no Teorema 5, chamamos de equação característica associada à recorrência $\mathrm{x}_{\mathrm{n}}(\mathrm{a}, \mathrm{q})=\sqrt[\mathrm{q}]{\mathrm{a}+\mathrm{x}_{\mathrm{n}-1}(\mathrm{a}, \mathrm{q})}, \operatorname{com} \mathrm{x}_{0}(\mathrm{a}, \mathrm{q})=\sqrt[q]{\mathrm{a}}$, a equação $\mathrm{x}^{\mathrm{q}}-\mathrm{x}-\mathrm{a}=0$ obtida após o processo de limite.

Exemplo 1. A equação característica associada a $x_{n}=\sqrt{2+x_{n-1}}, \operatorname{com~} x_{0}=\sqrt{2}$, é $x^{2}-x-2=0$. Mais geralmente, a equação quadrática $x^{2}-x-a=0$ é a equação característica da recorrência $\mathrm{x}_{\mathrm{n}}=\sqrt{\mathrm{a}+\mathrm{x}_{\mathrm{n}-1}}$, com valor inicial $\mathrm{x}_{0}=\sqrt{\mathrm{a}}$.

Como sabemos, não é uma tarefa fácil obter raízes da equação característica quando q $\geq 3$. Na próxima seção faremos um estudo dessa equação para $q \geq 3$, utilizando um método bastante simples.

Exemplo 2. Segue do Teorema 5 que a sequência $\sqrt[3]{6}, \sqrt[3]{6+\sqrt[3]{6}}, \cdots$, é convergente. Reescrevendo a sequência via recorrência temos $x_{n+1}=\sqrt[3]{6+x_{n}}$. Com isso, o limite procurado é dado pela raiz positiva $\mathrm{x}^{3}-\mathrm{x}-6=0$. Podemos checar facilmente que 2 é uma solução positiva dessa equação. Com isso,

$$
\sqrt[3]{6+\sqrt[3]{6+\sqrt[3]{6+\cdots}}}=2
$$

Exemplo 3. Segue do Teorema 5 também que a sequência $\sqrt[4]{14}, \sqrt[4]{14+\sqrt[4]{14}}, \cdots$, é convergente. Assim, a equação característica é $\mathrm{x}^{4}-\mathrm{x}-14=0$. Note que 2 é uma solução positiva dessa equação. Logo,

$$
\sqrt[4]{14+\sqrt[4]{14+\sqrt[4]{14+\cdots}}}=2
$$


Exemplo 4. De forma análoga, observando que 2 é solução das equações

$$
x^{5}-x-30=0 \text { e } x^{7}-x-126=0
$$

vemos que $\sqrt[5]{30+\sqrt[5]{30+\sqrt[5]{30+\cdots}}}=\sqrt[7]{126+\sqrt[7]{126+\sqrt[7]{126+\cdots}}}=2$

O que os exemplos 2, 3 e 4 têm em comum é o fato de 2 ser raiz da equação característica para $q=3,4,5$ e 7 . O que fizemos foi, fixado q, variar o argumento a de forma a garantir que 2 seja uma solução. Na sequência iremos sistematizar esse método.

\subsection{Interseção de gráficos}

A função $f_{q}: \mathbb{R} \rightarrow \mathbb{R}$ dada por $f_{q}(x)=x^{q}-x, q \geq 2$ inteiro positivo, desempenha um papel importante neste trabalho. Observe que a função $\mathrm{f}_{\mathrm{q}}(\mathrm{x})=\mathrm{x}^{\mathrm{q}}-\mathrm{x}$ em geral não é sobrejetora. Portanto, de forma a garantir os nossos resultados, restringimos o domínio. Devido à definição da sequência $x_{n}(a, q)$, estamos interessados em soluções positivas para a equação (6). Supondo $0<\mathrm{x} \leq 1$, a função $\mathrm{f}_{\mathrm{q}}$ assume apenas valores negativos, o que contraria nossos propósitos. Então vamos analisar a função $\mathrm{f}_{\mathrm{q}}(\mathrm{x})$ para valores de $\mathrm{x}>1$.

Teorema 6. Para todo inteiro $\mathrm{q} \geq 2$ a função $\mathrm{f}_{\mathrm{q}}:(1, \infty) \rightarrow(0, \infty)$ dada por $\mathrm{f}_{\mathrm{q}}(\mathrm{x})=\mathrm{x}^{\mathrm{q}}-\mathrm{x}$, é bijetora.

Demonstração. Note que, pelo Corolário 1, temos que para q $\geq 2$ inteiro, a equação

$$
\mathrm{x}^{\mathrm{q}}-\mathrm{x}-\mathrm{a}=0
$$

possui uma única solução positiva. Portanto, dado a $>0$, existe $x>1$ tal que $f_{q}(x)=x^{q}-x=a$ e concluímos que $\mathrm{f}_{\mathrm{q}}$ é sobrejetora para $\mathrm{q} \geq 2$.

Nesse momento, vamos verificar a injetividade de $\mathrm{f}_{\mathrm{q}}$. Sejam $\mathrm{x}_{1}, \mathrm{x}_{2} \in(1, \infty)$ com $\mathrm{x}_{1} \neq \mathrm{x}_{2}$. Suponhamos por absurdo que $\mathrm{f}_{\mathrm{q}}\left(\mathrm{x}_{1}\right)=\mathrm{f}_{\mathrm{q}}\left(\mathrm{x}_{2}\right) \Rightarrow \mathrm{x}_{1}^{\mathrm{q}}-\mathrm{x}_{1}=\mathrm{x}_{2}^{\mathrm{q}}-\mathrm{x}_{2}$. Isso implica

$$
\left(\mathrm{x}_{1}-\mathrm{x}_{2}\right)=\mathrm{x}_{1}^{\mathrm{q}}-\mathrm{x}_{2}^{\mathrm{q}}=\left(\mathrm{x}_{1}-\mathrm{x}_{2}\right)\left(\mathrm{x}_{1}^{\mathrm{q}-1}+\mathrm{x}_{1}^{\mathrm{q}-2} \mathrm{x}_{2}+\cdots+\mathrm{x}_{1} \mathrm{x}_{2}^{\mathrm{q}-2}+\mathrm{x}_{2}^{\mathrm{q}-1}\right)
$$

implicando

$$
\mathrm{x}_{1}^{\mathrm{q}-1}+\mathrm{x}_{1}^{\mathrm{q}-2} \mathrm{x}_{2}+\cdots+\mathrm{x}_{1} \mathrm{x}_{2}^{\mathrm{q}-2}+\mathrm{x}_{2}^{\mathrm{q}-1}=1,
$$

o que é um absurdo, uma vez que $\mathrm{x}_{1}, \mathrm{x}_{2}>1$ e $\mathrm{q} \geq 2$. Logo, $\mathrm{f}_{\mathrm{q}}\left(\mathrm{x}_{1}\right) \neq \mathrm{f}_{\mathrm{q}}\left(\mathrm{x}_{2}\right)$.

Observação 3. Uma outra maneira de demonstrar a injetividade de $\mathrm{f}_{\mathrm{q}}$ seria utilizando técnicas de Cálculo Diferencial. Mais precisamente, temos que a derivada $\mathrm{f}_{\mathrm{q}}^{\prime}(\mathrm{x})=\mathrm{qx}^{\mathrm{q}-1}-1>0$ para todo $\mathrm{q} \geq 2$ e $x>1$ e portanto $f_{q}$ é uma função injetiva.

Segue do Teorema 6 que, dado um número real a $>0$ e um inteiro q $\geq 2$, existe um único número real $\mathrm{x} \in(1, \infty)$ tal que $\mathrm{f}_{\mathrm{q}}(\mathrm{x})=\mathrm{a}$. Isto possibilita-nos empregar a técnica que passaremos a descrever.

Note que, geometricamente, toda reta horizontal acima do eixo das abcissas, dada pela função constante $\mathrm{h}_{\mathrm{q}}(\mathrm{x})=\mathrm{a}>0$, para cada número real a e inteiro $\mathrm{q} \geq 2$, intersecta o gráfico de $\mathrm{f}_{\mathrm{q}}(\mathrm{x})$ em um único ponto, digamos $\mathrm{x}_{0}$, ou seja, $\mathrm{a}=\mathrm{h}_{\mathrm{q}}\left(\mathrm{x}_{0}\right)=\mathrm{f}_{\mathrm{q}}\left(\mathrm{x}_{0}\right)$.

Então, a ideia central desse método consiste em encontrar, dentre tais pontos, o ponto de interseção adequado no seguinte sentido. Queremos encontrar um ponto de intersecção $\mathrm{x}_{0}$ tal que a equação 
$\mathrm{f}_{\mathrm{q}}(\mathrm{x})=\mathrm{h}_{\mathrm{q}}\left(\mathrm{x}_{0}\right)$ seja de fácil resolução. Graficamente, $\mathrm{h}_{\mathrm{q}}(\mathrm{x})=\mathrm{h}_{\mathrm{q}}\left(\mathrm{x}_{0}\right)$ é uma reta horizontal que intersecta o gráfico de $\mathrm{f}_{\mathrm{q}}(\mathrm{x})$ no ponto $\left(\mathrm{x}_{0}, \mathrm{f}_{\mathrm{q}}\left(\mathrm{x}_{0}\right)\right)$.

Agora suponha que tenhamos encontrado tal ponto $\mathrm{x}_{0}$, e consequentemente $\mathrm{h}_{\mathrm{q}}\left(\mathrm{x}_{0}\right)$. Seja $\mathrm{x}_{1}$ a única solução de $\mathrm{f}_{\mathrm{q}}(\mathrm{x})=\mathrm{h}_{\mathrm{q}}\left(\mathrm{x}_{0}\right)$, isto é, $\mathrm{x}_{1}$ satisfaz a equação $\mathrm{x}^{\mathrm{q}}-\mathrm{x}-\mathrm{h}_{\mathrm{q}}\left(\mathrm{x}_{0}\right)=0$. Então o Teorema 5 garante que $\mathrm{x}_{1}$ é o limite da sequência dada pela recorrência $\mathrm{x}_{\mathrm{n}}\left(\mathrm{h}_{\mathrm{q}}\left(\mathrm{x}_{0}\right)\right)=\sqrt[q]{\mathrm{h}_{\mathrm{q}}\left(\mathrm{x}_{0}\right)+\mathrm{x}_{\mathrm{n}-1}\left(\mathrm{~h}_{\mathrm{q}}\left(\mathrm{x}_{0}\right)\right)}$, com valor inicial $\sqrt[q]{\mathrm{h}_{\mathrm{q}}\left(\mathrm{x}_{0}\right)}$.

Para determinarmos o ponto $\mathrm{x}_{0}$, e consequentemente $\mathrm{h}_{\mathrm{q}}\left(\mathrm{x}_{0}\right)$, fazemos interseção do gráfico de $\mathrm{f}_{\mathrm{q}}(\mathrm{x})$ com o gráfico de uma função auxiliar dada. As funções auxiliares que nos permitem encontrar $\mathrm{x}_{0}$ facilmente são $g_{1}(x)=x, g_{2}(x)=r x$ e $g_{3}(x)=r x$, em que $r$ é um número real positivo. Note que $\mathrm{g}_{1}$ é um caso particular de $\mathrm{g}_{2}$, mas procedemos em casos separados porque assim percebemos a evolução das ideias no sentido de obter resultados cada vez mais gerais. Note que não podemos obter $g_{1}(x)$ e $g_{2}(x)$ a partir de $g_{3}(x)$ pois estamos considerando $q \geq 2$. No entanto, como veremos, $\mathrm{g}_{2}$ e $\mathrm{g}_{3}$ nos conduzem ao mesmo resultado.

Caso 01: $\mathrm{g}_{1}(\mathrm{x})=\mathrm{x}$.

Neste caso, estamos interessados nos pontos fixos de $\mathrm{f}_{\mathrm{q}}$, isto é, queremos encontrar todos os pontos $\mathrm{x}$ em $(1, \infty)$ tais que $\mathrm{f}_{\mathrm{q}}(\mathrm{x})=\mathrm{x}$.

Lema 4. Para todo inteiro $\mathrm{q} \geq 2$, a função $\mathrm{f}_{\mathrm{q}}$ tem um único ponto fixo.

Demonstração. De fato, $\mathrm{f}_{\mathrm{q}}(\mathrm{x})=\mathrm{x}$ se, e somente se, $\mathrm{x}^{\mathrm{q}}-\mathrm{x}=\mathrm{x}$ e por sua vez isso ocorre se, e somente se, $\mathrm{x}^{\mathrm{q}}-2 \mathrm{x}=0$, o que é equivalente $\mathrm{a} \mathrm{x}\left(\mathrm{x}^{\mathrm{q}-1}-2\right)=0$. A última equação possui duas soluções, a saber $\mathrm{x}_{1}=0$ e $\mathrm{x}=\sqrt[\mathrm{q}-1]{2}$. Como $0 \notin(1, \infty)$, temos somente uma solução e esta é o ponto fixo de $\mathrm{f}_{\mathrm{q}}$.

Seguindo a discussão acima do Lema 4 , tomamos $\mathrm{x}_{0}=\sqrt[\mathrm{q}-1]{2}$ e $\mathrm{h}_{\mathrm{q}}\left(\mathrm{x}_{0}\right)=\mathrm{x}_{0}$. Como podemos ver, $\mathrm{a}$ equação $\mathrm{f}_{\mathrm{q}}(\mathrm{x})=\mathrm{h}_{\mathrm{q}}\left(\mathrm{x}_{0}\right)$ é de fácil resolução, já que o ponto fixo de $\mathrm{f}_{\mathrm{q}}$ é a solução. Analise as figuras abaixo. A Figura 1 é referente a q ímpar, e a Figura 2 é quando q é um número par.

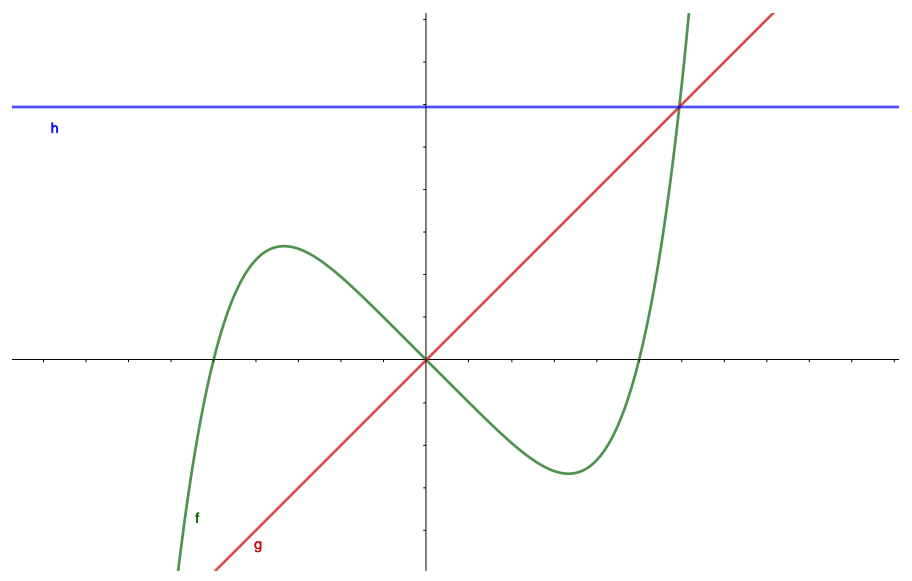

Figura 1: $f_{5}(x)=x^{5}-x, g_{1}(x)=x$ e $h_{5}(\sqrt[4]{2})=\sqrt[4]{2}$ 


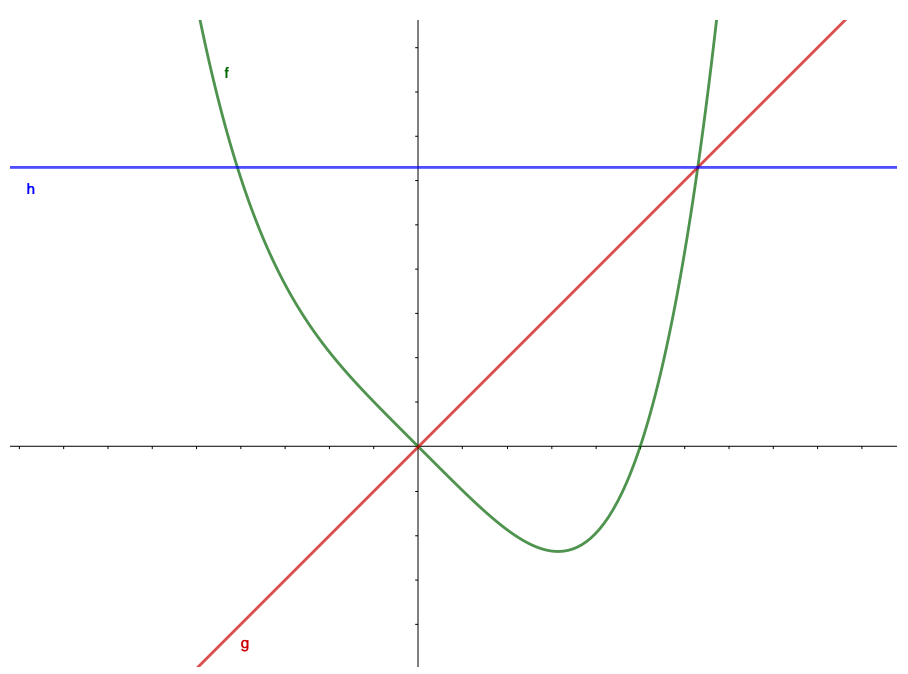

Figura 2: $\mathrm{f}_{4}(\mathrm{x})=\mathrm{x}^{4}-\mathrm{x}, \mathrm{g}_{1}(\mathrm{x})=\mathrm{x} \mathrm{e} \mathrm{h}_{4}(\sqrt[3]{2})=\sqrt[3]{2}$

Com isso, segue do Teorema 5 e do Lema 4, nosso próximo resultado, o qual nos fornece os pontos fixos das sequências de radicais encaixados generalizadas que tratamos aqui e os quais são a solução da Questão 1.

Teorema 7. Para todo inteiro $\mathrm{q} \geq 2$ vale

$$
\sqrt[q]{q-1 \sqrt{2}+\sqrt[q]{\frac{q-1}{2}+\sqrt[q]{q-1} \sqrt{2}+\cdots}}=\sqrt[q-1]{2}
$$

Observação 4. Note que fazendo $q=2$, obtemos $\sqrt{2+\sqrt{2+\sqrt{2+\cdots}}}=2$, ou seja, recaímos no Teorema 2.

Exemplo 5. Tomando $q=3$, obtemos $\sqrt[3]{\sqrt{2}+\sqrt[3]{\sqrt{2}+\sqrt[3]{\sqrt{2}+\cdots}}}=\sqrt{2}$

Observação 5. O Teorema 7 pode ser obtido também da seguinte maneira. Queremos encontrar um valor $\mathrm{h}(\mathrm{q})$ para o limite da sequência de modo que $\mathrm{h}(\mathrm{q})^{\mathrm{q}}=\mathrm{h}(\mathrm{q})+\mathrm{h}(\mathrm{q})$. Isto leva-nos a $\mathrm{h}(\mathrm{q})=\sqrt[\mathrm{q}-1]{2}$. Mas a continuidade do artigo deixará claro por que utilizamos o método da interseção dos gráficos.

Caso 02: $\mathrm{g}_{2}(\mathrm{x})=\mathrm{rx}$, onde $\mathrm{r} \geq 0$.

A fim de encontrarmos outras raízes positivas da equação característica, modificamos um pouco a técnica de pontos fixos obtendo uma versão mais geral. Para isso, resolvemos a equação $f_{q}(x)=r x$ para algum $r \in \mathbb{R}_{+}$em vez de $f_{q}(x)=x$. Isso leva-nos à equação

$$
x^{q}-(r+1) x=0
$$

que, por sua vez, possui $\mathrm{x}=\sqrt[\mathrm{q}-1]{\mathrm{r}+1}$ como raiz. Assim, temos o seguinte resultado:

Lema 5. Para todo $\mathrm{q} \geq 2, \mathrm{x}=\sqrt[\mathrm{q}-1]{\mathrm{r}+1}$ é raiz da equação $\mathrm{x}^{\mathrm{q}}-\mathrm{x}-\mathrm{r}^{\mathrm{q}-1} \sqrt{\mathrm{r}+1}=0$. 
Demonstração. Somando e subtraindo rx e rearranjando os termos na equação acima obtemos

$$
\begin{aligned}
\mathrm{x}^{\mathrm{q}}-\mathrm{x}-\mathrm{r}^{\mathrm{q}-1} \sqrt{\mathrm{r}+1} & =\mathrm{x}^{\mathrm{q}}-\mathrm{x}-\mathrm{rx}+\mathrm{rx}-\mathrm{r}^{\mathrm{q}-1} \sqrt{\mathrm{r}+1} \\
& =\mathrm{x}^{\mathrm{q}}-(\mathrm{r}+1) \mathrm{x}+\mathrm{rx}-\mathrm{r}^{\mathrm{q}-1} \sqrt{\mathrm{r}+1}
\end{aligned}
$$

Note que, $\sqrt[q-1]{\mathrm{r}+1}-(\mathrm{r}+1) \sqrt[\mathrm{q}-1]{\mathrm{r}+1}+\mathrm{r}^{\mathrm{q}-1} \sqrt{\mathrm{r}+1}-\mathrm{r}^{\mathrm{q}-1} \sqrt{\mathrm{r}+1}=0$, pois $\sqrt[\mathrm{q}-1]{\mathrm{r}+1}-(\mathrm{r}+1)^{\mathrm{q}-1} \sqrt[1]{\mathrm{r}+1}=0$ pela equação (7) e obviamente $\mathrm{r}^{\mathrm{q}-1} \sqrt{\mathrm{r}+1}-\mathrm{r}^{\mathrm{q}-1} \sqrt{\mathrm{r}+1}=0$. Portanto, $\sqrt[\mathrm{q}-1]{\mathrm{r}+1}-\sqrt[q]{\mathrm{q}-1} \sqrt{\mathrm{r}+1}-\mathrm{r}^{\mathrm{q}-1} \sqrt{\mathrm{r}+1}=0$.

Nesse caso, $\mathrm{x}_{0}=\sqrt[\mathrm{q}-1]{\mathrm{r}+1}$ e $\mathrm{h}_{\mathrm{q}}\left(\mathrm{x}_{0}\right)=\mathrm{rx}_{0}$. Assim, segue do Lema 5 que $\mathrm{f}_{\mathrm{q}}(\mathrm{x})=\mathrm{h}_{\mathrm{q}}\left(\mathrm{x}_{0}\right)$ tem solução $\mathrm{x}_{0}$. Veja a Figura 3 que se refere a $\mathrm{q}$ par, e a Figura 4 referente a q ímpar.

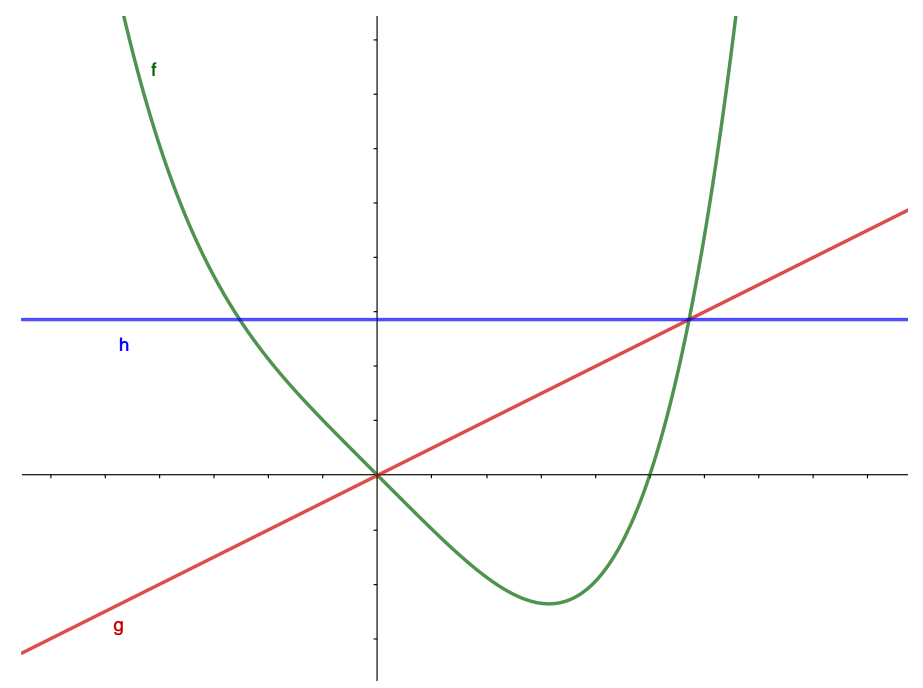

Figura 3: $\mathrm{f}_{4}(\mathrm{x})=\mathrm{x}^{4}-\mathrm{x}, \mathrm{g}_{1}(\mathrm{x})=\frac{1}{2} \mathrm{x}$ e $\mathrm{h}_{4}\left(\sqrt[3]{\frac{3}{2}}\right)=\frac{1}{2} \sqrt[3]{\frac{3}{2}}$. 


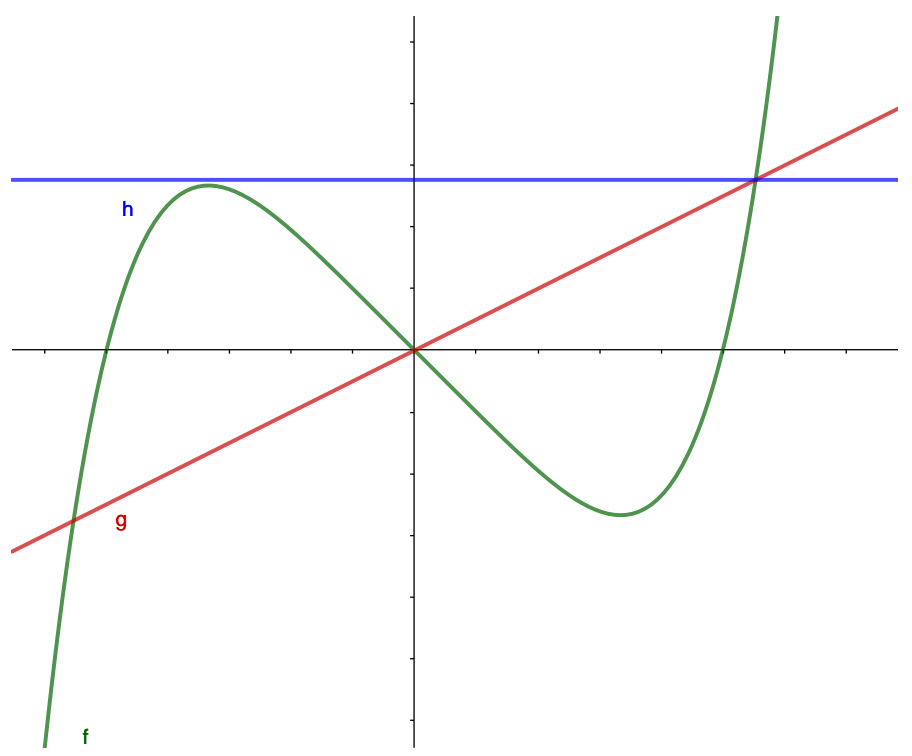

Figura 4: $f_{5}(x)=x^{5}-x, g_{2}(x)=\frac{1}{2} x$ e $h_{5}\left(\sqrt[4]{\frac{3}{2}}\right)=\frac{1}{2} \sqrt[4]{\frac{3}{2}}$

Do Lema 5 e do Teorema 5 decorre o resultado seguinte.

Teorema 8. Para todo inteiro $\mathrm{q} \geq 2$ e $\mathrm{r}>0$ vale

$$
\sqrt[q]{\mathrm{r}^{\mathrm{q}-1} \sqrt{\mathrm{r}+1}+\sqrt[q]{\mathrm{r}^{\mathrm{q}-1} \sqrt{\mathrm{r}+1}+\sqrt[q]{\mathrm{r}^{\mathrm{q}-1} \sqrt{\mathrm{r}+1}+\cdots}}}=\sqrt[\mathrm{q}-1]{\mathrm{r}+1}
$$

As Observações 6 e 7 abaixo, refletem a importância deste Teorema.

Observação 6. Tomando $\mathrm{q}=2$ e $\mathrm{r}=1$ obtemos $\sqrt{2+\sqrt{2+\sqrt{2+\cdots}}}=2$. Ou seja, de fato generalizamos o Teorema 2 bem como o Teorema 3 para índices mais gerais do radical e também argumentos mais gerais.

Observação 7. Tomando $\mathrm{q}=2$, temos $\sqrt{\mathrm{r}(\mathrm{r}+1)+\sqrt{\mathrm{r}(\mathrm{r}+1)+\sqrt{\mathrm{r}(\mathrm{r}+1)+\cdots}}}=\mathrm{r}+1$. Ou seja, recaímos no Teorema 4 para índices mais gerais do radical. A dificuldade encontrada em tentar generalizar o Teorema 4 via técnica de [13] é o fato que para $q \geq 3$ as expressões para as raízes das equações $\mathrm{x}^{\mathrm{q}}-\mathrm{x}-\mathrm{a}=0$ ficam bastante complicadas. Todavia, a técnica aqui empregada permitiu generalizar esse Teorema.

Exemplo 6. Tomando $q=3$, temos $\sqrt[3]{\mathrm{r} \sqrt{\mathrm{r}+1}+\sqrt[3]{\mathrm{r} \sqrt{\mathrm{r}+1}+\sqrt[3]{\mathrm{r} \sqrt{\mathrm{r}+1}+\cdots}}}=\sqrt{\mathrm{r}+1}$. Assim,

$$
\begin{aligned}
& 3=\sqrt[3]{24+\sqrt[3]{24+\sqrt[3]{24+\sqrt[3]{24+\cdots}}}}, \quad \text { onde } r=8 \\
& 4=\sqrt[3]{60+\sqrt[3]{60+\sqrt[3]{60+\sqrt[3]{60+\cdots}}}}, \text { onde } r=15
\end{aligned}
$$


Exemplo 7. Tomando $q=4$, temos $\sqrt[4]{\mathrm{r} \sqrt[3]{\mathrm{r}+1}+\sqrt[4]{\mathrm{r} \sqrt[3]{\mathrm{r}+1}+\sqrt[4]{\mathrm{r} \sqrt[3]{\mathrm{r}+1}+\cdots}}}=\sqrt[3]{\mathrm{r}+1}$. Assim,

$$
\begin{aligned}
& 2=\sqrt[4]{14+\sqrt[4]{14+\sqrt{14+\sqrt[4]{14+\cdots}}}}, \quad \text { onde } r=7 \\
& 3=\sqrt[4]{78+\sqrt[4]{78+\sqrt[4]{78+\sqrt[4]{78+\cdots}}}}, \text { onde } r=26
\end{aligned}
$$

Observação 8. Note que, dado $\mathrm{q} \geq 2$ e $\mathrm{r}=\frac{\mathrm{x}}{\mathrm{y}}>0$ uma fração arbitrária, temos que

$$
\sqrt[q-1]{\left(\frac{x}{y}\right)^{1}+\left(\frac{x}{y}\right)^{0}}=\sqrt[q]{\sqrt[q-1]{\left(\frac{x}{y}\right)^{q}+\left(\frac{x}{y}\right)^{q-1}}+\sqrt[q]{\sqrt[q-1]{\left(\frac{x}{y}\right)^{q}+\left(\frac{x}{y}\right)^{q-1}}+\sqrt[q]{q^{-1} \sqrt{\left(\frac{x}{y}\right)^{q}+\left(\frac{x}{y}\right)^{q-1}}+\cdots}}}
$$

O leitor pode verificar que uma aplicação direta do Teorema 8 é dada pela identidade

$$
\frac{a}{b}=\sqrt[q]{\sqrt[q-1]{\left(\frac{a^{q^{-1}}-b^{q^{-1}}}{b^{q^{-1}}}\right)^{q}+\left(\frac{a^{q^{-1}}-b^{q^{-1}}}{b^{q^{-1}}}\right)^{q^{-1}}}+\sqrt[q]{\sqrt[q-1]{\left(\frac{a^{q^{-1}}-b^{q^{-1}}}{b^{q^{-1}}}\right)^{q}+\left(\frac{a^{q^{-1}}-b^{q^{-1}}}{b^{q^{-1}}}\right)^{q^{-1}}}+\cdots}}
$$

onde $\mathrm{a}>\mathrm{b}$ são inteiros positivos arbitrários. Além disso é interessante atribuir valores para a e b obtendo vários exemplos de como expressar frações como limite de uma sequência de radicais encaixados.

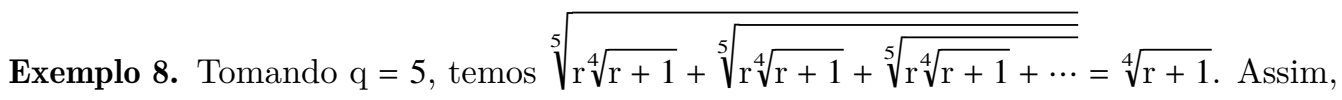

$$
\begin{aligned}
& \sqrt{2}=\sqrt[5]{3 \sqrt{2}+\sqrt[5]{3 \sqrt{2}+\sqrt[5]{3 \sqrt{2}+\cdots}}}, \text { onde } r=3 \\
& \sqrt{3}=\sqrt[5]{8 \sqrt{3}+\sqrt[5]{8 \sqrt{3}+\sqrt[5]{8 \sqrt{3}+\cdots}}}, \text { onde } r=8
\end{aligned}
$$

Caso 03: $\mathrm{g}_{3}(\mathrm{x})=\mathrm{rx}^{\mathrm{q}}$, onde $0<\mathrm{r}<1$.

Nesse caso estamos interessados em resolver a equação $\mathrm{f}_{\mathrm{q}}(\mathrm{x})=\mathrm{rx}^{\mathrm{q}}$ para algum $\mathrm{r} \in \mathbb{R}_{+}$. Isso leva-nos à equação

$$
(1-\mathrm{r}) \mathrm{x}^{\mathrm{q}}-\mathrm{x}=0
$$

que possui $\mathrm{x}=1 / \sqrt[\mathrm{q}-1]{1-\mathrm{r}}$, como raiz. Assim, temos o

Lema 6. Para todo inteiro $\mathrm{q} \geq 2$ e $0<\mathrm{r}<1, \mathrm{x}_{0}=\frac{1}{\sqrt[4-1]{1-\mathrm{r}}}$ é raiz da equação $\mathrm{x}^{\mathrm{q}}-\mathrm{x}-\frac{\mathrm{r}}{\sqrt[\mathrm{q}^{-1}]{(1-\mathrm{r})^{\mathrm{q}}}}=0$.

Demonstração. Somando e subtraindo $\mathrm{rx}^{\mathrm{q}}$ e rearranjando os termos na equação acima obtemos

$$
\begin{aligned}
x^{q}-x-\frac{r}{\sqrt[q-1]{(1-r)^{q}}} & =x^{q}-x-r x^{q}+r x^{q}-\frac{r}{\sqrt[q-1]{(1-r)^{q}}} \\
& =(1-r) x^{q}-x+r x^{q}-\frac{r}{\sqrt[q-1]{(1-r)^{q}}} .
\end{aligned}
$$


Segue da equação $(8)$ que $\mathrm{x}_{0}(\mathrm{r})=\frac{1}{\mathrm{q}-\sqrt[1]{1-\mathrm{r}}} \operatorname{satisfaz}(1-\mathrm{r}) \mathrm{x}^{\mathrm{q}}-\mathrm{x}=0$ e é fácil ver que satisfaz também $\mathrm{rx}^{\mathrm{q}}-\frac{\mathrm{r}}{\sqrt[\mathrm{q}-1]{(1-\mathrm{r})^{\mathrm{q}}}}=0$. Logo, satisfaz $(1-\mathrm{r}) \mathrm{x}^{\mathrm{q}}-\mathrm{x}+\mathrm{rx}^{\mathrm{q}}-\frac{\mathrm{r}}{\sqrt[\mathrm{q}-1]{(1-\mathrm{r})^{\mathrm{q}}}}=0$ e, consequentemente, $\mathrm{x}^{\mathrm{q}}-\mathrm{x}-\frac{\mathrm{r}}{\sqrt[\mathrm{q}-1]{(1-\mathrm{r})^{\mathrm{q}}}}=0$.

Portanto, se tomarmos $\mathrm{x}_{0}=\frac{1}{\sqrt[\mathrm{q}-1]{1-\mathrm{r}}}$ e $\mathrm{h}_{\mathrm{q}}\left(\mathrm{x}_{0}\right)=\frac{\mathrm{r}}{\sqrt[\mathrm{q}-1 \sqrt{(1-\mathrm{r})^{\mathrm{q}}}]{ }}$, então a equação $\mathrm{f}_{\mathrm{q}}(\mathrm{x})=\mathrm{h}_{\mathrm{q}}\left(\mathrm{x}_{0}\right)$ é de fácil resolução, veja Figura 5.

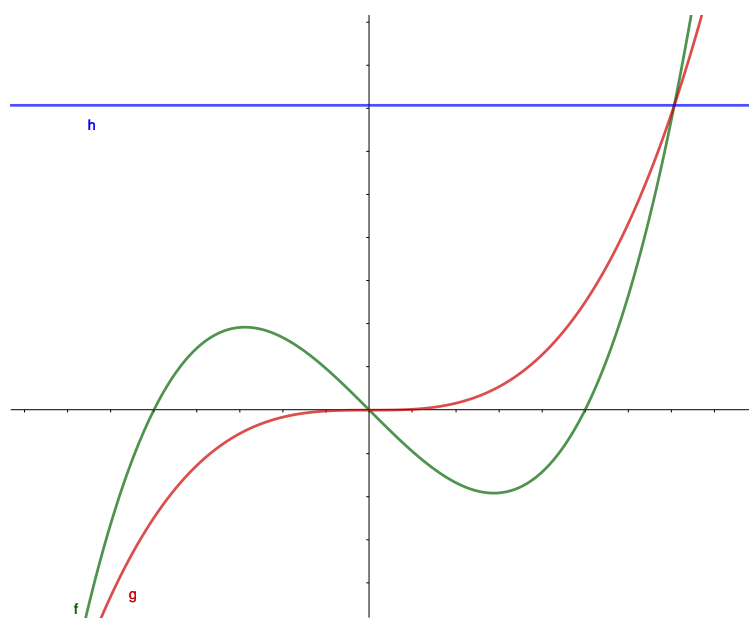

Figura 5: $\mathrm{f}_{3}(\mathrm{x})=\mathrm{x}^{3}-\mathrm{x}, \mathrm{g}_{3}(\mathrm{x})=\frac{1}{2} \mathrm{x}^{3}$ e $\mathrm{h}_{3}(\sqrt{2})=\sqrt{2}$.

Portanto, segue do Lema 6 e do Teorema 5 o seguinte resultado.

Teorema 9. Para todo inteiro $\mathrm{q} \geq 2$ e $0<\mathrm{r}<1$ vale

$$
\sqrt[q]{\frac{r}{\sqrt[q-1]{(1-r)^{q}}}+\sqrt[q]{\frac{r}{\sqrt[q-1]{(1-r)^{q}}}+\sqrt[q]{\frac{r}{\sqrt[q-1]{(1-r)^{q}}}+\cdots}}}=\frac{1}{\sqrt[q-1]{1-r}} .
$$

Observação 9. Veja que

$$
\frac{\mathrm{r}}{\sqrt[\mathrm{q}-1]{(1-\mathrm{r})^{\mathrm{q}}}}=\sqrt[\mathrm{q}-1]{\frac{\mathrm{r}^{\mathrm{q}-1}}{(1-\mathrm{r})^{\mathrm{q}}}}=\sqrt[\mathrm{q}-1]{\frac{\mathrm{r}^{\mathrm{q}-1}}{(1-\mathrm{r})^{\mathrm{q}-1}} \frac{1}{(1-\mathrm{r})}}=\frac{\mathrm{r}}{1-\mathrm{r}} \sqrt[\mathrm{q}-1]{\frac{1}{1-\mathrm{r}}} .
$$

Faça a mudança $s=r /(1-r)$. Note que quando $r$ percorre o intervalo $(0,1)$ vemos que $s$ percorre $(0, \infty)$. Note também que $1-r=1 /(s+1)$. Então substituindo na Equação (9) obtemos

$$
\sqrt[q]{s \sqrt[q-1]{s+1}+\sqrt[q]{s^{q-1} \sqrt{s+1}+\sqrt[q]{s^{q-1} \sqrt{s+1}+\cdots}}}=\sqrt[q-1]{s+1},
$$

o que é precisamente o conteúdo do Teorema 8. Com isso, as funções auxiliares $\mathrm{g}_{2}(\mathrm{x})=\mathrm{rx}$ e $\mathrm{g}_{3}(\mathrm{x})=\mathrm{rx}^{\mathrm{q}}$ apesar de fornecerem uma maneira fácil de encontrar a solução de $\mathrm{f}_{\mathrm{q}}(\mathrm{x})=\mathrm{h}_{\mathrm{q}}\left(\mathrm{x}_{0}\right)$, conduzem-nos ao mesmo resultado. 


\section{Considerações finais}

Existe uma lista bastante extensa na literatura de trabalhos que lidam com sequências infinitas de radicais encaixados das mais variadas formas. Os pontos centrais abordados nesses trabalhos são a convergência da sequência e a possibilidade de escrever o limite de forma explícita. Acrescentamos a essa lista este trabalho em que apresentamos a classe de sequência generalizada de radicais encaixados da forma $\left(x_{n}(a, q)\right)_{n}=(\sqrt[q]{a}, \sqrt[q]{a+\sqrt[q]{a}}, \cdots)$. Por meio dos Teoremas 5,7 e 8 foi mostrado que tal sequência é convergente, que o limite pode ser expresso explicitamente e caracterizamos seus pontos fixos. Nosso próximo passo, é estudar a sequência de radicais encaixados da forma $\left(x_{n}(a, b, q)\right)_{n}=(\sqrt[q]{a}, \sqrt[q]{a \pm b \sqrt[q]{a}}, \sqrt[q]{a \pm b \sqrt[q]{a \pm b \sqrt[q]{a}} \cdots)}$, que passa a depender de um novo parâmetro b, empregando as técnicas desenvolvidas aqui.

\section{Agradecimentos}

Valdemiro Carlos dos Santos Silva Filho agradece à Capes pelo apoio através da concessão de bolsa durante o curso de mestrado profissional ProfMat-UFT Arraias.

\section{Referências}

[1] Barbosa, J. L. M. Geometria Euclidiana Plana, Ed 11, SBM, 2012.

[2] Barbosa, R.; Feitosa, S. Obmep - Banco de Questões 2016, Rio de Janeiro, Impa, 2016.

[3] Barbosa, R.; Feitosa, S. Obmep - Banco de Questões 2018, Rio de Janeiro, Impa, 2018.

[4] Beckmann, P. A history of $\pi$, 3rd ed. Boulder, CO: The Golem Press. p. 94-95, 1971.

[5] Chang, M-L.; Chang, C-C. "Evaluation of Pi by Nested Radicals", Mathematics Magazine, Vol. $89, \mathrm{n}^{\circ} 5,2016$.

[6] Herschfeld, A. "On Infinite Radicals", The American Mathematical Monthly, Vol. 42, n7, pp.419-429, 1935.

[7] Lima, E. L. Curso de Análise . Vol 1, décima segunda edição, Impa, Rio de Janeiro, 2006.

[8] McGuffin, M. Nested Radicals And Other Infinitely Recursive Expressions, for The Pure Math Club University of Waterloo, 1998. Disponível em: < http : //www.dgp.toronto.edu/ mjmcguff/math/nestedRadicals.pdf >. Acesso em: 08 de fevereiro de 2020.

[9] Kanigel, R. The Man Who Knew Infinity: A Life of the Genius Ramanujan, Washington Square Press, 1991.

[10] Nyblom, M. A. "More Nested Square Roots of 2". The American Mathematical Monthly, Vol. $112, \mathrm{n}^{\circ} 9$, pp. 822-825, 2005.

[11] Rao, K.S.; Berghe, G.V. "On an entry of Ramanujan in his Notebooks: a nested roots expansion", Journal of Computational and Applied Mathematics, Vol 173, 2005.

[12] Servi, L. D. "Nested Square Roots of 2", The American Mathematical Monthly, Vol. 110, n4, pp.326-330, 2003.

[13] Zimmerman, S.; Ho, C. "On Infinitely Nested Radicals", Mathematics Magazine, Vol. 81, n¹, pp.3-15, 2008. 
Élis Gardel Mesquita Universidade Federal do Tocantins <elisgardel@mail.uft.edu.br>

Thiago R. Cavalcante Universidade Federal do Tocantins <thiago.cavalcante@mail.uft.edu.br>

Valdemiro Carlos S. S. Filho Escola Municipal Ottomar Schwengber $<$ valdemiro_carlos@yahoo.com>

Recebido: 03/04/2020

Publicado: 02/03/2021 